\title{
Induction of multiple myeloma cancer stem cell apoptosis using conjugated anti-ABCG2 antibody with epirubicin-loaded microbubbles
}

\author{
Fangfang Shi ${ }^{1,2}$, Miao Li ${ }^{2}$, Jing Wang ${ }^{3}$, Di Wu ${ }^{2,3}$, Meng Pan ${ }^{2}$, Mei Guo ${ }^{1}$ and Jun Dou ${ }^{2 *}$
}

\begin{abstract}
Background: Multiple myeloma (MM) currently remains largely incurable. Cancer stem cells (CSCS) are believed to be responsible for drug resistance and eventual relapse. In this study, we exploited a novel agent to evaluate its inhibitory effect on MM CSCS.

Methods: Epirubicin (EPI)-loaded lipid microbubbles (MBs) conjugated with anti-ABCG2 monoclonal antibody (EPI$\mathrm{MBs}+\mathrm{mAb}$ ) were developed and their effect on MM $138^{-} \mathrm{CD} 34^{-}$CSCs isolated from human MM RPMI 8226 cell line plus ultrasound exposure in vitro and in vivo in a nonobese diabetic/severe combined immunodeficient mouse model were assessed.

Results: $\mathrm{EPI}-\mathrm{MBs}+\mathrm{mAb}$ combined with ultrasound led to a significant decrease in the clone formation ability and the mitochondrial membrane potential along with an increase in MM CSC apoptosis. Moreover, treatment with EPI-MBs + mAb with ultrasound exposure remarkably inhibited the growth of MM CSC-derived tumors in xenograft nonobese diabetic/severe combined immunodeficient mice compared with a single agent or EPI-MBs + mAb without ultrasound exposure. The inhibitive efficacy was also correlated with an increased expression of caspase-3, Bax, and TUNEL and decreased expressions of PCNA, BCl-2, and CD31.

Conclusions: Our findings reveal that the EPI-MBs + mAb combined with therapeutic ultrasound may confer an effective approach for treatment of MM by induction of an apoptotic pathway in MM CSCS.
\end{abstract}

Keywords: Multiple myeloma, Cancer stem cells, Epirubicin, Microbubble, Ultrasound

\section{Background}

Multiple myeloma (MM) is a plasma cell malignancy characterized by the growth of MM cells in the bone marrow, and it is still incurable. Although hematopoietic stem cell transplantation and advances in chemotherapy, such as proteasome inhibitors and immunomodulatory drugs, have significantly achieved lasting remission and increased survival rates in patients with $\mathrm{MM}$, most patients eventually relapse [1-3]. Cancer stem cells (CSCs), which show the features of self-renewal, multidirectional differentiation, and multidrug resistance, are thought to

\footnotetext{
* Correspondence: njdoujun@seu.edu.cn

${ }^{2}$ Department of Pathogenic Biology and Immunology, School of Medicine,

Southeast University, 87\# Ding Jiaqiao Rd., Nanjing 210009, China

Full list of author information is available at the end of the article
}

be the major cause of tumor chemoresistance and recurrence. MM treatment failure has demonstrated that current therapeutic agents are not enough to eradicate MM CSCs [4-7].

Epirubicin (EPI) is one of the anthracycline drugs commonly used for therapy in MM, breast, sarcoma, and many other malignant tumors. Since EPI can be distributed both in tumor and normal tissue when injected intravenously it shows various adverse reactions, including digestive tract toxicity, myelosuppression, functional lesions in the liver and kidney, and so on $[8,9]$. To reduce the side effects and enhance the local drug concentration in MM chemotherapy, we hypothesize that it may be feasible to use an ultrasound-targeted microbubble destruction (UTMD) technique combined with a

(C) The Author(s). 2018 Open Access This article is distributed under the terms of the Creative Commons Attribution 4.0 International License (http://creativecommons.org/licenses/by/4.0/), which permits unrestricted use, distribution, and 
specific antibody as a drug targeting MM CSCs to increase anti-MM efficacy [10].

Microbubbles (MBs) are a rapid-development drugdelivery system with many advantages, including high security and drug-loading capability using the different prepared methods. The biological effect of UTMD on MBs might enhance the capability of MB drug delivery. For example, EPI was released from the targeting MBs when ultrasound exposure was applied to tumors, which enhanced the EPI concentration in local tumor tissue and reduced the cytotoxicity of EPI to the host body. Therefore, drug-loaded MBs conjugated with antibody in combination with UTMD appears to be a promising strategy for the treatment of tumors [11-13].

The high drug-efflux capability of MM CSCs is likely to be a major cause of drug resistance in MM. ABCG2 is one of the ATP-binding cassette $(\mathrm{ABC})$ transporters that represents the family of transmembrane proteins. It is known that ABCG2 is highly expressed in MM CSCs, showing a strong activity to efflux cytotoxic compounds [14-17]. We previously demonstrated that the combination of anti-ABCG2 monoclonal antibody (mAb) and paclitaxel iron oxide nanoparticles resulted in improving anti-MM CSC efficacy. In the present study, to reduce both drug resistance and the adverse reaction to EPI, we exploited a novel agent using EPI-loaded lipid MBs conjugated with anti-ABCG2 mAb to assess its effect on MM $138^{-} \mathrm{CD} 34^{-}$CSCs under ultrasound exposure.

Here, we show that the combination of EPI-MBs + $\mathrm{mAb}$ with therapeutic ultrasound could achieve a significant inhibition of $\mathrm{MM} \mathrm{CD} 138^{-} \mathrm{CD} 34^{-} \mathrm{CSC}$ clone formation and induction of CSC apoptosis in vitro, as well as a reduction in $\mathrm{MM} \mathrm{CD} 138^{-} \mathrm{CD} 34^{-} \mathrm{CSC}$-derived tumor growth in nonobese diabetic/severe combined immunodeficient (NOD/SCID) mice compared with using EPI alone or EPI-MBs + mAb without ultrasound exposure. This synergy of the combination of EPI-MBs + mAb with UTMD may be considered for further preclinical trials in the treatment of drug-resistant MM.

\section{Methods}

\section{Cell Line and Mice}

Human MM RPMI 8226 cells were purchased from the Cell Bank of the Chinese Academy of Sciences (Beijing, China). Cells were cultured in complete medium consisting of RPMI 1640, $2 \mathrm{mM} \mathrm{L-glutamine,} 100 \mathrm{U} / \mathrm{mL}$ penicillin, $100 \mu \mathrm{g} / \mathrm{mL}$ streptomycin, and $10 \%$ fetal bovine serum at $37{ }^{\circ} \mathrm{C}$ in a humidified incubator containing $5 \%$ $\mathrm{CO}_{2}$. NOD/SCID mice at 6 weeks of age (17-18 g weight) were purchased from Beijing Weitong Lihua Experimental Animal Technology Co., Ltd., China. Mice were maintained in a pathogen-free facility that has a 12-h light/dark cycle and relative humidity ranged from $40 \%$ to $50 \%$ at $24{ }^{\circ} \mathrm{C}$. All the animal experiments were performed in compliance with the Guidelines of the Animal Research Ethics Board of Southeast University. This ethics board also approved the animal studies.

\section{Preparation of EPI-MBs $+\mathrm{mAb}$}

The EPI-MBs + mAb were prepared as described in our previously published paper [18]. CY7.5 labeled EPI-MBs and EPI-MBs + mAb were obtained by adding proper CY7.5 (Lumiprobe, LLC) to chloroform in the preparation $[19,20]$.

\section{Isolation of $\mathrm{MM} \mathrm{CD} 138^{-} \mathrm{CD} 34^{-} \mathrm{CSC}$}

$\mathrm{CD} 138^{-} \mathrm{CD} 34^{-}$cells were isolated from the human MM RPMI 8226 cell line by a magnetic activated cell sorting method (Miltenyi Biotec, Gladbach, Germany) following our previous study protocol $[16,18,21]$. We named $\mathrm{CD} 138^{-} \mathrm{CD} 34^{-}$cells as MM CSCs.

\section{EPI uptake efficiency of MM CD138- CD34- CSCs}

$\mathrm{MM} \mathrm{CD} 138^{-} \mathrm{CD} 34^{-} \mathrm{CSCs}\left(1 \times 10^{6}\right)$ in a vial were respectively incubated with phosphate-buffered saline (PBS) , EPI, or EPI-MBs + mAb for $30 \mathrm{~min}$. The EPI concentration was $10 \mu \mathrm{g} / \mathrm{mL}$ in the vial, and the vial was placed upside down to maximize the cell-MB interaction. Ultrasound exposure was performed for $40 \mathrm{~s}$ on vials at $0.5 \mathrm{~W} / \mathrm{cm}^{2}$ [22]. The vial was cultured at $37^{\circ} \mathrm{C}$ in an incubator containing $5 \% \mathrm{CO}_{2}$ for an additional $30 \mathrm{~min}$. $\mathrm{MM} \mathrm{CD} 138^{-} \mathrm{CD} 34^{-}$ CSCs in the vial were washed three times with PBS to remove $\mathrm{MBs}+\mathrm{mAb}$ and excess EPI (1000 $\mathrm{rpm} / 8 \mathrm{~min})$, and stained with 4,6-diamidino-2-phenylindole (DAPI) for 10 mins, and then washed with PBS three times (2000 rpm/ $5 \mathrm{~min}$ ). The fluorescence intensity of coal maceral in MM $\mathrm{CD} 138^{-} \mathrm{CD} 34^{-} \mathrm{CSCs}$ was observed under a confocal fluorescence microscope [23]. The process was performed in the dark at room temperature.

\section{Colony formation assay}

MM CD $138^{-}$CD $34^{-}$CSCs $\left(1 \times 10^{6}\right)$ were treated with different agents following our previous protocol [18]. One hundred single-cell suspension cells were resuspended in $0.8 \mathrm{~mL}$ conditioned medium $(\mathrm{CM})$ containing $0.3 \%$ low melting temperature agarose (Promega, USA) and were plated in triplicate on 24-well plates over a base layer of $0.8 \mathrm{~mL} \mathrm{CM}$ containing $0.6 \%$ low melting temperature agarose. The plates were incubated for $10-$ 14 days until colonies were formed. Colony diameters larger than $75 \mu \mathrm{m}$ or colonies containing more than 50 cells were then counted as one positive colony according to previous reports $[4,24]$.

\section{Analysis of mitochondrial membrane potential and cell cycle}

For the mitochondrial membrane potential, $1 \times 10^{6} \mathrm{MM}$ CSCs treated with the different agents were washed 
twice with PBS and then resuspended in $500 \mu \mathrm{L} \mathrm{JC-1}$ buffer for $15-20 \mathrm{~min}$ in incubators at $37{ }^{\circ} \mathrm{C}$ with $5 \%$ $\mathrm{CO}_{2}$. Cells were washed in $1 \times 500 \mu \mathrm{L}$ incubation buffer twice $(2000 \mathrm{rpm} / 5 \mathrm{~min})$ and were resuspended in incubation buffer and analyzed by flow cytometry (FCM) $[18,25]$.

For the cell cycle analysis, $1 \times 10^{6}$ MM CSCs treated with the different agents were fixed overnight with $70 \%$ $(\mathrm{w} / \mathrm{v})$ ice-cold ethanol. Cells were resuspended in $1 \mathrm{~mL}$ PBS containing $40 \mu \mathrm{g} / \mathrm{mL}$ Annexin V/propidium iodide (PI) and $500 \mathrm{U} / \mathrm{mL}$ RNase A. Following incubation for $30 \mathrm{~min}$ in the dark at room temperature, cells were analyzed by FCM using the system modfit software. The PI fluorescence signal peak versus the integral was used to discriminate G2-M cells from G0-G1 doublets [26]. The cell cycle and JC-1 apoptosis detection kits were obtained from Keygen Biotech (China).

\section{Detection of EPI-MBs + $\mathrm{mAb}$ binding to MM tissues and ultraphonic echo intensity}

MM CD138 ${ }^{-}$CD34- CSCs $\left(1 \times 10^{6} ; 100 \mu \mathrm{L}\right)$ were mixed with matrigel (BD Biosciences; $100 \mu \mathrm{L}$ in volume) and injected subcutaneously (s.c.) into the right dorsal side of NOD/SCID mice. When the grafted s.c. tumor volume reached $0.8-1.0 \mathrm{~cm}$, the mice were divided into EPI-MBs + mAb (Anti-ABCG2 mAb; Cell Signaling Corporation) and EPI-MBs groups (three per group). In the EPI-MBs + mAb group, $0.2 \mathrm{~mL}$ EPI-MBs + mAb (MBs $3 \times 10^{9} / \mathrm{mL}$, EPI $0.5 \mathrm{mg} / \mathrm{mL}$ ) was injected into the mice through the tail vein. The same amount of EPI-MBs were injected accordingly in the other groups. Ultrasound imaging of subcutaneous tumors was performed before the injection of EPI-MBs + mAb and $30 \mathrm{~min}$ after the injection with a color Doppler diagnostic apparatus (My Lab Twice) equipped with a 5-8 MHz broadband linear transducer (mechanical index 0.06). Ultrasound issued by the same transducer (mechanical index 0.4) was then performed for $3 \mathrm{~min}$ on tumors [27]. Thirty minutes later, two mice in each group were executed, and the subcutaneous tumors were removed for frozen sectioning. The distribution of EPI-MBs + mAb and EPI$\mathrm{MBs}$ in the MM tissues was observed under a light microscope.

\section{Treatment of MM-bearing mice}

The human RPMI 8226 MM CSC xenograft NOD/SCID mice were randomly divided into PBS, EPI, EPI-MBs + $\mathrm{mAb}$, and EPI-MBs + mAb + ultrasound (US) groups (six per group). Then, $200 \mu \mathrm{L}$ of different agents including PBS, EPI $(5 \mu \mathrm{g} / \mathrm{kg})$, and EPI-MBs + mAb (EPI $5 \mu \mathrm{g} / \mathrm{kg}+$ $\mathrm{mAb} 0.5 \mu \mathrm{g} / \mathrm{g}$ ) were s.c. injected into the mice after MM-bearing mice had been established for a total of six times and once every 3 days [28]. In the EPI-MBs + $\mathrm{mAb}+\mathrm{US}$ group, UTMD was applied $30 \mathrm{~min}$ after the injection on tumors with a mechanical index of 0.4 for 3 min [22]. The tumor growth in NOD/SCID mice was monitored once 3 days for tumor volume by measuring two perpendicular tumor diameters from each mouse using calipers. The survival time was also observed in the remaining mice.

\section{Western blot}

Tumor tissues were collected from each mouse and homogenized for detection of P-p65 and P-IкB $\alpha$ expression [29]. According to the manufacturer's protocol, the proteins were separated using sodium dodecyl sulfatepolyacrylamide gel electrophoresis. The membrane was blocked with the buffer containing $10 \%$ fat-free dry milk; the rabbit antihuman P-p65, P-IKB $\alpha$, and $\beta$-actin antibodies were respectively used as the primary antibody for $2 \mathrm{~h}$, and the membrane was rinsed for $5 \mathrm{~min}$ with an antibody wash solution three times before adding a secondary horseradish peroxidase-conjugated anti-rabbit antibody for $1 \mathrm{~h}$ at room temperature. The following steps were performed according to Kit's protocol [18].

\section{Immunohistochemistry and histopathology of tumor tissues}

Immunostaining was performed as previously reported [30]. Briefly, 5- $\mu \mathrm{m}$ thin formalin-fixed and paraffinembedded tumor sections were incubated with the rabbit anti-mouse/human caspase-3, Bax, TUNEL, PCNA (proliferating cell nuclear antigen), $\mathrm{Bcl}-2$, and $\mathrm{CD} 31$, respectively, overnight at $4{ }^{\circ} \mathrm{C}$. The antibody concentration was 1:600. The samples were then labeled with horseradish peroxidase-conjugated streptavidin (Invitrogen) and the chromogenic reaction was developed using the Liquid DAB Substrate Pack according to the manufacturer's instructions. The stained cells from random and nonoverlapping fields were counted under a magnification of $\times 100$ or $\times 400$. Meanwhile, the sections were stained with hematoxylin and eosin for microscopic examination. All sections were observed in a blinded fashion and were photographed using a $400 \times$ normal light microscope.

\section{Statistical analysis}

The data were plotted as mean $\pm \mathrm{SD}$ and analyzed for statistical significance by two-tailed paired Student's $t$ test or repeated measures analysis of variance (ANOVA). $P$ values less than 0.05 were considered statistically significant. Analyses were performed with the SPSS 19.0 software package.

\section{Results}

Analysis of MM CD $138^{-} \mathrm{CD} 34^{-} \mathrm{CSC}$ uptake of EPI

EPI-loaded MBs with conjugated anti-ABCG2 antibody $(\mathrm{EPI}-\mathrm{MBs}+\mathrm{mAb})$ were prepared as described in our 
previous work [18]. To show the EPI uptake efficiency of MM CD138 ${ }^{-} \mathrm{CD} 34^{-} \mathrm{CSCs}$, we detected the fluorescence intensity in $\mathrm{MM} \mathrm{CD} 138^{-} \mathrm{CD} 34^{-} \mathrm{CSCs}$ by a confocal fluorescence microscopy. Figure 1a shows that $\mathrm{MM}$ CD138 ${ }^{-}$CD $34^{-}$CSCs showed the highest fluorescence intensity among the three tested groups when CSCs were incubated with EPI-MBs + mAb combined with UTMD, indicating that more EPI (shown in red in the figure) accumulated in $\mathrm{MM} \mathrm{CD} 138^{-} \mathrm{CD} 34^{-}$CSCs, which was statistically significant compared with the EPI group $(P<0.01)$ or PBS (control) group $(P<0.001)$. Although EPI was partly taken up by MM CD $138^{-} \mathrm{CD} 34^{-}$CSCs, the efficiency of EPI uptake was significant lower with no $\mathrm{MBs}+\mathrm{mAb}$ and ultrasound exposure than that of EPI-MBs + mAb combined with ultrasound exposure, as shown in Fig. 1b. The results suggested that the EPI-
MBs + mAb combined with UTMD could effectively target MM CD138 ${ }^{-} \mathrm{CD} 34^{-} \mathrm{CSCs}$ and enhance EPI accumulation in MM CSCs in vitro.

\section{Effect of EPI-MBs + mAb combined with UTMD on MM CSCs}

First, we observed the effect of EPI-MBs + mAb combined with UTMD on MM CSCs in vitro. Figure 2a shows that the combined EPI-MBs + mAb with UTMD inhibited the clonogenic capability of MM CSCs in soft agar media. The clone formation rate was significantly lower in the EPI-MBs + mAb combined with UTMD group than that of the EPI-MBs + mAb without using UTMD group $(4.3 \pm 1.21 \%$ versus $27.2 \pm 0.98 \%, P<0.01)$, the EPI group $(4.3 \pm 1.21 \%$ versus $16.8 \pm 1.15 \%, P<0.05)$, or the PBS group $(4.3 \pm 1.21 \%$ versus $32.5 \pm 4.54 \%, P<0.01)$

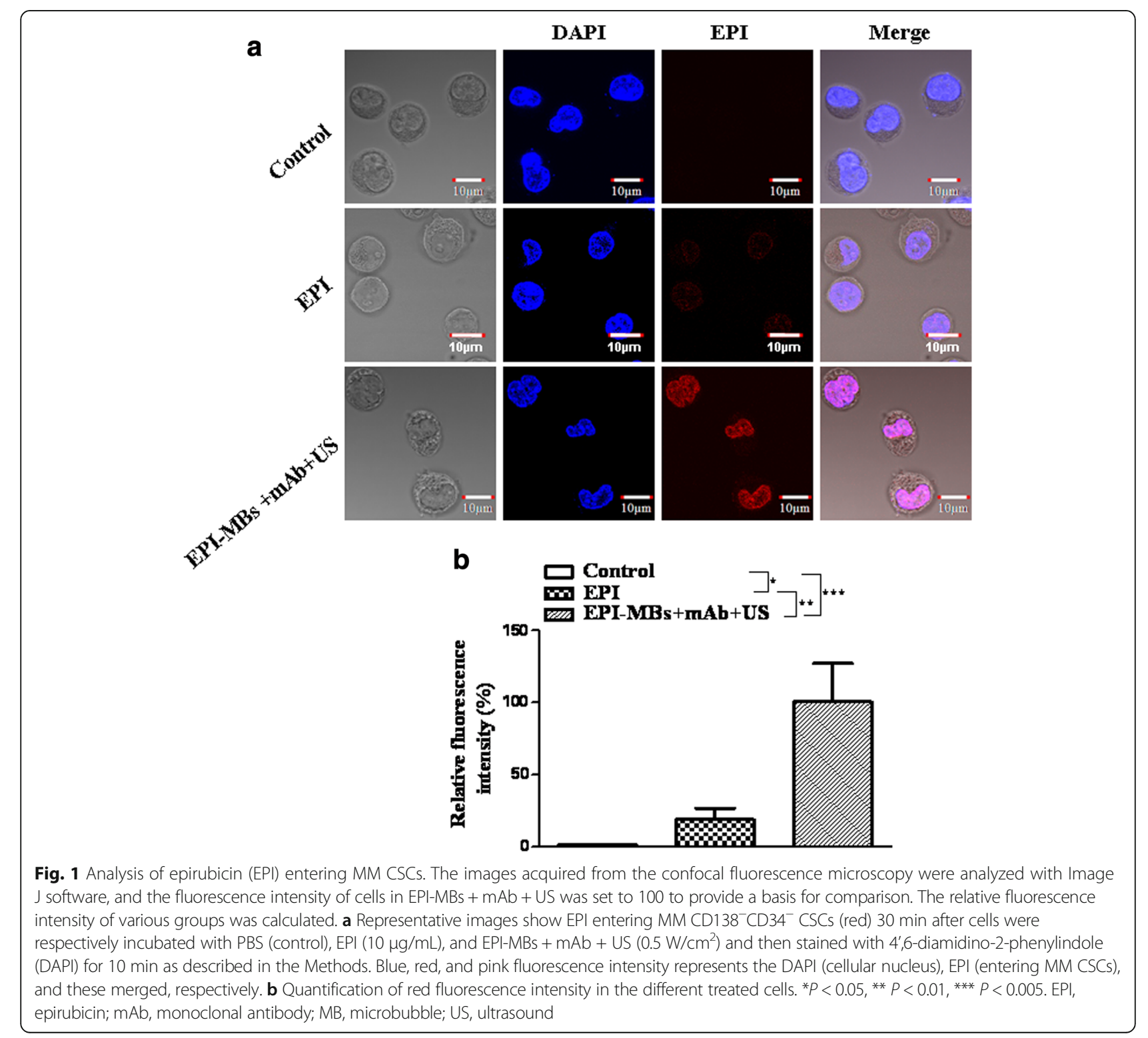




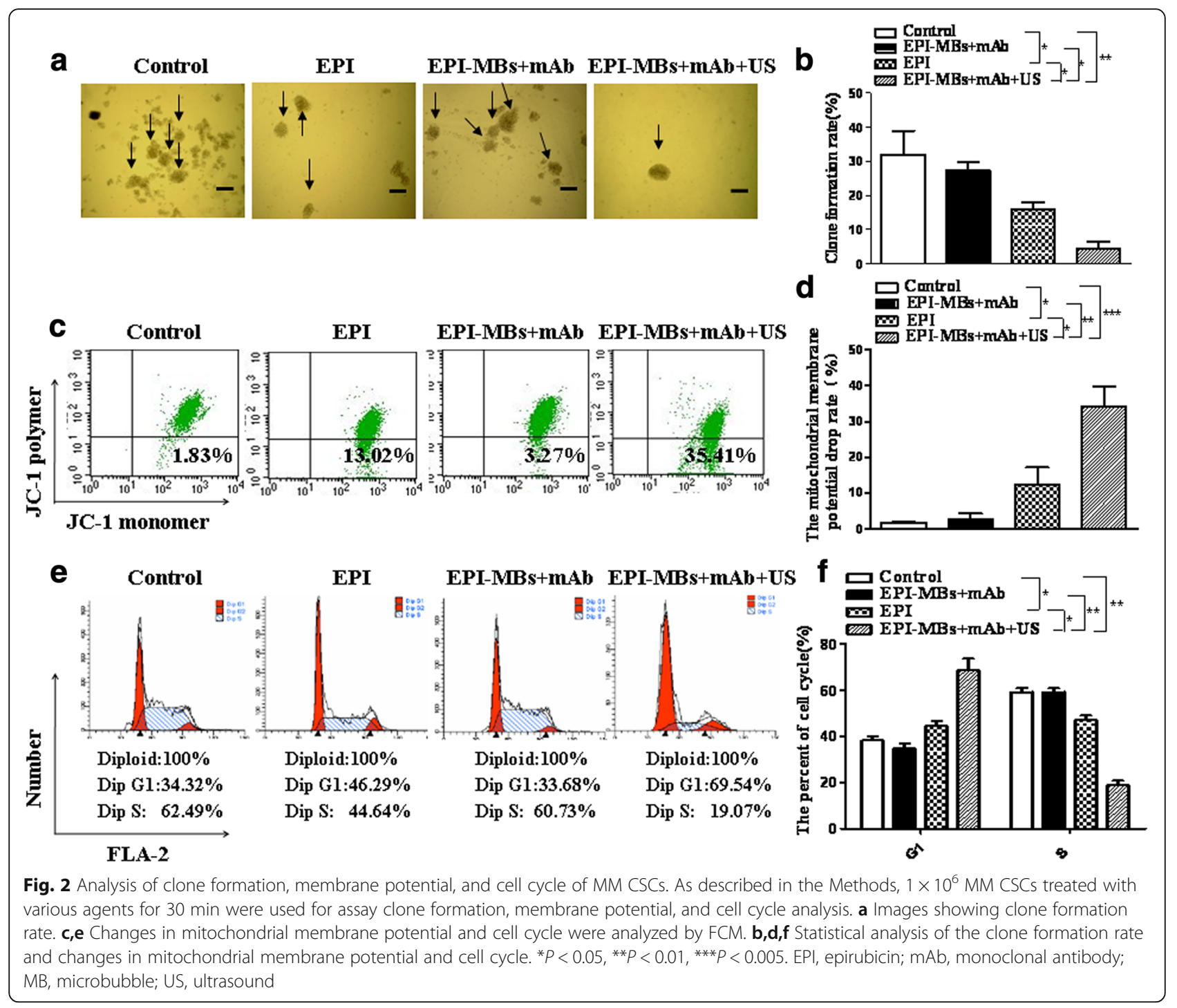

(Fig. 2b). Similar efficacy was also found in the mitochondrial membrane potential change (Fig. 2c), which showed a significantly increased mitochondrial membrane potential drop rate in the MM CSCs in the EPI-MBs + mAb combined with UTMD group compared with the EPIMBs + mAb without UTMD group (35.41 \pm 5.53 versus $3.27 \pm 1.01 \%, P<0.01)$, EPI group $(35.41 \pm 5.53$ versus $13.02 \pm 4.80 \%, P<0.05)$, or PBS group $(35.41 \pm 5.53$ versus $1.83 \pm 0.27 \%, P<0.01)$. There were significant differences between the EPI-MBs + mAb combined with UTMD and the EPI-MBs + mAb groups and between the EPI-MBs + mAb combined with UTMD and the EPI groups (Fig. 2d).

Subsequently, we analyzed the effect of different agents on the cell cycle and apoptosis of MM CSCs by FCM. The data presented in Fig. 2e shows that the highest percentage of G1 phase cell count was in the EPI-MBs + mAb combined with UTMD group, which was statistically significant compared with the EPI-MBs + mAb without UTMD group (69.54 versus $33.68 \%, P<0.01$ ), EPI group (69.54 versus $46.29 \%, P$ $<0.05$ ), or PBS group (69.54 versus $34.32 \%, P<0.01$ ). In contrast to the $\mathrm{G} 1$ phase, the cell count in the $S$ phase was lower in the EPI-MBs + mAb combined with UTMD group than that in the EPI-MBs + mAb without UTMD group $(19.07$ versus $60.73 \%, P<0.01)$ , EPI group (19.07 versus 44.64\%, $P<0.05$ ), or PBS group (19.07 versus $62.49 \%, P<0.01)$ (Fig. 2f). All the data from the in vitro experiments demonstrated that the EPI-MBs + mAb combined with US exposure had a significant efficacy on inhibition of clone formation and induction of MM CSC apoptosis, suggesting the UTMD destroys the MBs and releases EPI from the MBs to the site of MM CSCs, and promotes EPI to easily enter cells through perforations in CSCs. 
Analysis of EPI-MBs + mAb-targeted binding to MM tissues

To show whether the EPI-MBs + mAb could target binding to MM tissues, we first established the MM-bearing mouse model. Figure 3a shows images of MM-bearing mice (Fig. 3a, left) 34 days after $1 \times 10^{6} \mathrm{MM} \mathrm{CD} 138^{-} \mathrm{CD} 34^{-}$cells were injected s.c. into the right dorsal side in NOD/SCID mice, but no tumor (Fig. 3a, right) was found in mice injected with $1 \times 10^{6}$ non-MM CD $138^{-} \mathrm{CD} 34^{-}$cells. Figure $3 \mathrm{~b}$ depicts the dynamic state curve of tumor growth in mice, suggesting the MM CD138 ${ }^{-} \mathrm{CD} 34^{-}$cells have CSC features such as self-renewal and tumorigenicity.

Next, we injected EPI-MBs + mAb or EPI-MBs into the MM-bearing mice through the tail vein. The images 30 min after injection show that a lot of MBs were located in the MM tissues of mice injected with EPI-MBs + mAb (Fig. 3c); however, only a few MBs were found in mice injected with EPI-MBs, which was statistically significant $(P<0.01$; Fig. $3 d)$. The results suggested that EPI-MBs + $\mathrm{mAb}$ could directly target binding to MM tissues owing to the specific anti-ABCG2 monoclonal antibody.

Further, we evaluated the ultraphonic echo intensity in the tumor tissues by ultrasonic testing. Representative images in Fig. 3e show the stronger ultraphonic echo intensity in the EPI-MBs + mAb group than in the EPIMBs group or control group, which was statistically significant $(P<0.05$; Fig. 3f). These data further supported that the ability to bind to MM tissue for EPI-MBs + mAb plus UTMD was better than that of EPI-MBs plus UTMD without $\mathrm{mAb}$ in the treatment of MM in vivo.

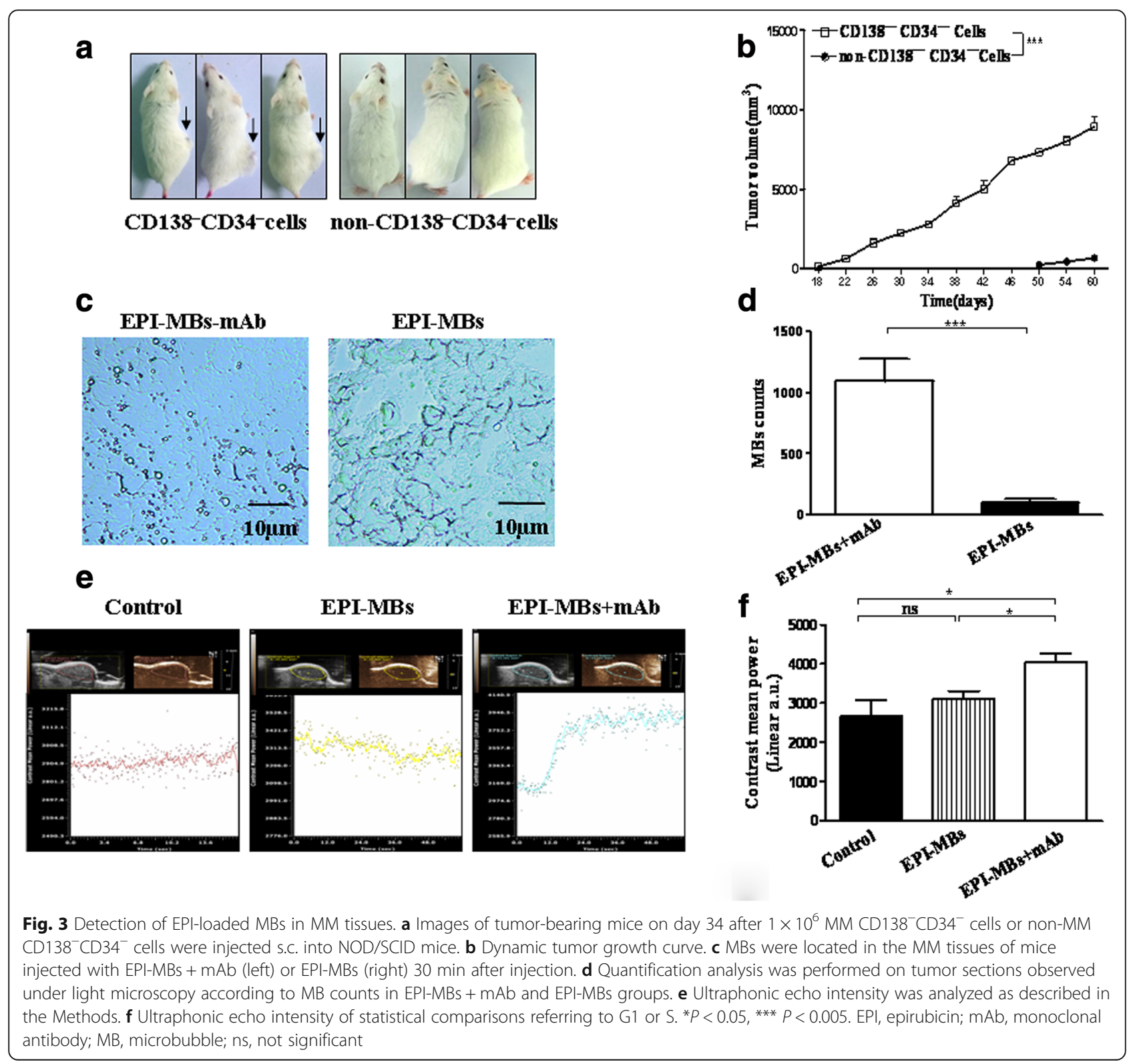


Evaluation of the effect of EPI-MBs + $\mathrm{mAb}$ combined with UTMD on MM-bearing mice

Since EPI-MBs + mAb could directly target the local MM tissue in mice, we sought to evaluate whether this targeted binding ability would effectively inhibit tumor growth in the MM CSC xenograft mouse model. The data presented in Fig. 4a show that all treated mice developed smaller tumors than did control mice without treatment but, in the EPI-MBs + mAb combined with UTMD group, the tumor volumes were significant smaller than in the EPI group $(P<0.01)$ or in the EPIMBs $+\mathrm{mAb}$ without US group $(P<0.05)$. In addition, mice treated with EPI-MBs $+\mathrm{mAb}$ combined with UTMD showed longer survival time than mice treated with the other agents in this study (Fig. 4b). There was a significant difference between the EPI-MBs + mAb combined with UTMD group and the EPI-MBs + mAb group $(P<0.05)$, between the EPI-MBs + mAb combined with UTMD group and the EPI group $(P<0.01)$, and between the EPI-MBs + mAb combined with UTMD group and the control group $(P<0.01)$.

Tumor histopathologic section images in Fig. 4c show that the tumor cells were distributed sparsely, and that some necrotic or apoptotic tumor cells were more noticeable in tumor tissues of mice treated with EPI-MBs $+\mathrm{mAb}$ combined with UTMD than those in mice treated with a single agent or combination agent without UTMD, which was statistically significant $(P<0.05$ or $P<0.01$; (Fig. 4d).

\section{Detection of apoptosis pathway-related molecule expression in tumor tissues}

To understand the anti-MM mechanisms of EPI-MBs + mAb combined with therapeutic ultrasound, we analyzed the related molecule expression in tumor tissues from mice using immunohistochemistry assay. Figure 5a, e shows the expression of Caspase-3, Bax, TUNEL, PCNA, Bcl-2, and CD31. We found that the tumor cells from the mice treated with EPI-MBs + mAb combined with UTMD significantly increased the staining of Caspase-3, Bax, and TUNEL, and remarkably decreased the staining of PCNA, Bcl-2, and CD31, compared to those in the tumor cells from the mice treated with EPI$\mathrm{MBs}+\mathrm{mAb}$, or EPI, or PBS control $(P<0.05$ or $P<0.01$ or $P<0.005$; Fig. $5 \mathrm{~b}-\mathrm{d}, \mathrm{f}-\mathrm{h})$. Meanwhile, the expression of P-p65 and P-IкB $\alpha$ as analyzed by Western blot (Fig. 5i) was also increased in the EPI-MBs + mAb combined with UTMD group, which was statistically significant

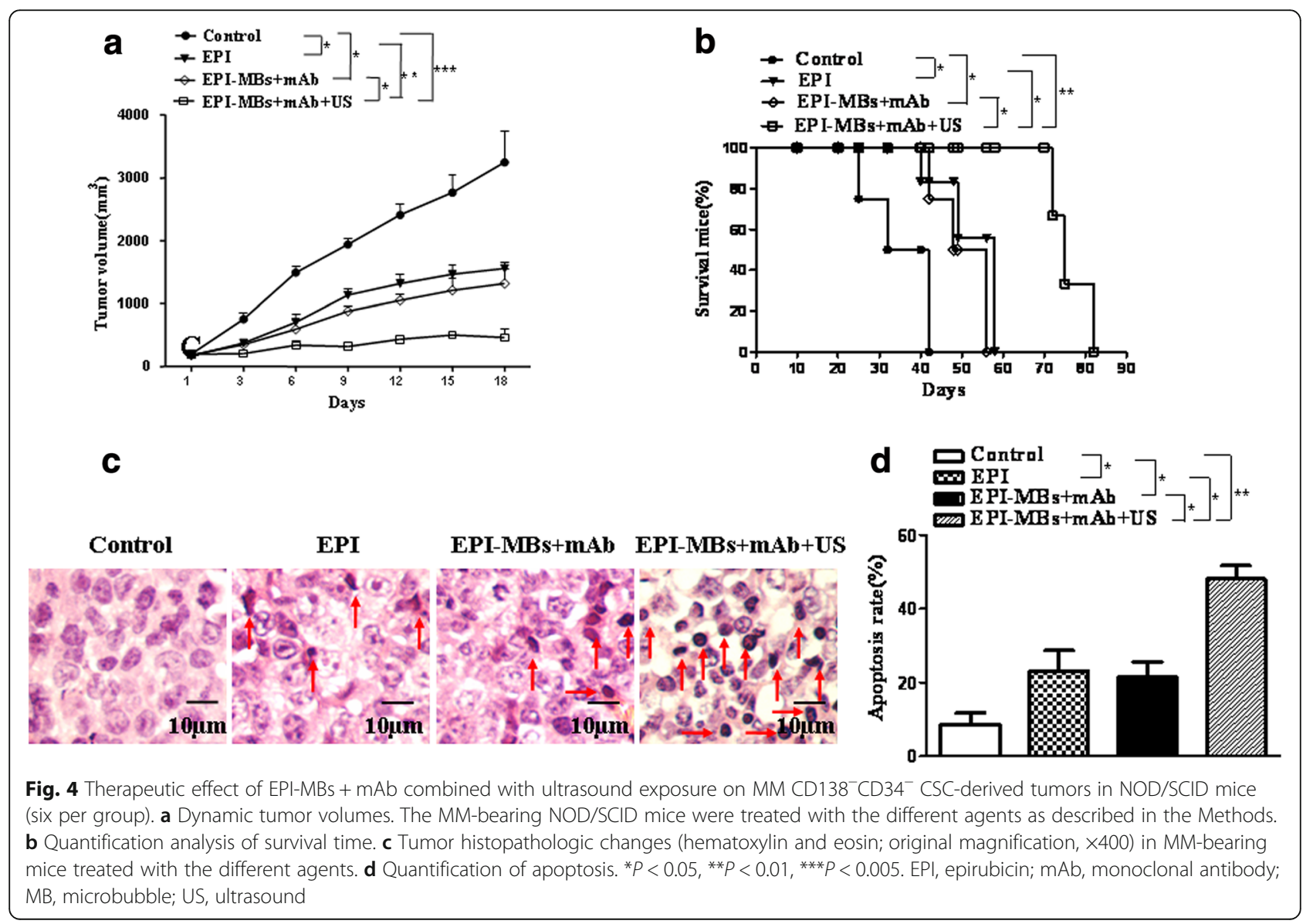



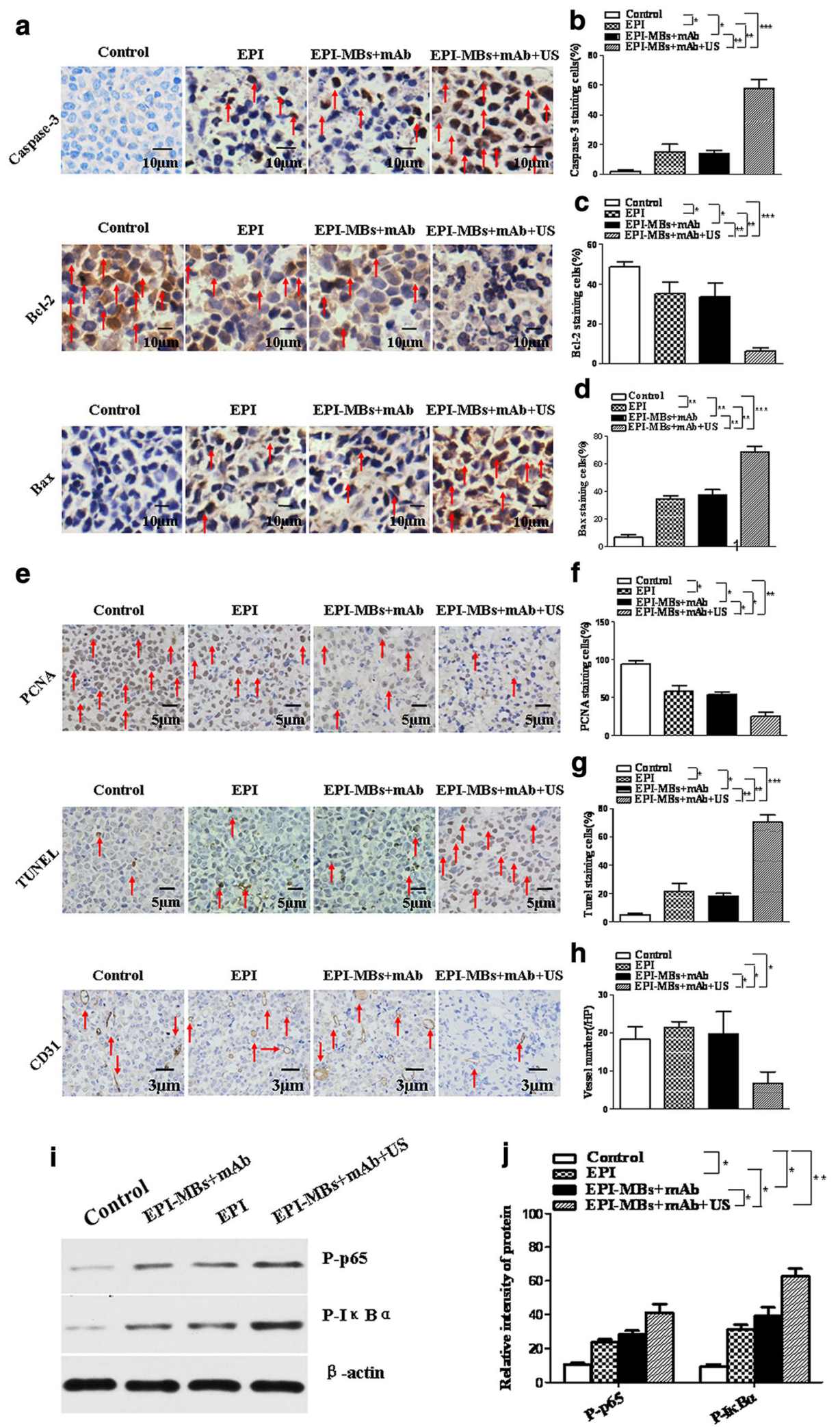

Fig. 5 (See legend on next page.) 
(See figure on previous page.)

Fig. 5 Expression of apoptosis-related molecules in tumor tissues analyzed with immunohistochemistry and Western blot. a,e Images of apoptosis-related molecule expression, including Caspase-3, Bax, TUNEL, PCNA, BCl-2, and CD31 analyzed with immunohistochemistry (a, original magnification $\times 400$; $\mathbf{e}$, original magnification $\times 100$ ). $\mathbf{b}-\mathbf{d}$,f-h Q $\mathbf{h}$ antification analysis of Caspase-3, Bax, TUNEL, PCNA, BCl-2, and CD31 expression. i Expression of P-p65and P-IkBa analyzed by Western blot. $\mathbf{j}$ Semiquantification analysis of molecular expression. ${ }^{*} P<0.05,{ }^{* *} P<0.01$. EPI, epirubicin; mAb, monoclonal antibody; MB, microbubble; US, ultrasound

compared with the EPI-MBs $+\mathrm{mAb}(P<0.05)$, EPI $(P$ $<0.05)$, and PBS groups $(P<0.01)$ (Fig. 5j).

\section{Discussion}

MM is a terminally differentiated plasma cell malignancy for which no consistently curative treatment exists. The molecular mechanisms underlying MM chemoresistance remain elusive. One possible explanation is that CSCs appear to be relatively resistant to standard anticancer therapies by co-opting intrinsic defense mechanisms of normal stem cell, such as quiescence, efflux pumps, and detoxifying enzymes [31, 32]. Therefore, there is a continued and unmet need to expand the therapeutic arsenal against this disease [24, 33]. Emerging evidence supports the view that targeting MM CSCs may increase the therapeutic effectiveness against MM [34]. In this regard, we focused on a combination of a conventional drug against MM with targeted ABCG2 ability as well as a UTMD technique to design an exciting therapeutic strategy for the treatment of the refractory disease in MM-bearing NOD/SCID mice.

To assess the effect of the therapeutic strategy on CD138 ${ }^{-}$CD $34^{-}$CSCs isolated from the human MM RPMI8226 cell line, we examined the capability for clonogenic formation and induction of apoptosis of MM CSCs in vitro. From the results of the colony formation assay, we found that the EPI-MBs + mAb combined with UTMD treated MM CSCs showed the lowest colony count among the four groups. It is known that the clone formation assay in the soft agar can be used to measure the ability of cells to cross tissue barriers and to measure the cell invasion, and that the cloning efficiency represents the cellular proliferative and self-renewal ability that is correlated positively with the disease stages of MM, plasma cell leukemia, or advanced MM [1, 24, 35]. Therefore, the data presented in our soft agar clone assay suggested that the EPI-MBs + mAb combined with UTMD could effectively inhibit the proliferation of MM CSCs, and this was further supported by the apoptosis and cell cycle assays. These results indicated that MM CSC apoptosis and cell cycle blocking were significantly higher in the EPI-MBs + mAb combined with UTMD group compared with any other groups.

Furthermore, the in vivo therapeutic results for EPIMBs + mAb combined with UTMD revealed stronger efficacy for inhibiting MM CSC-derived tumor growth and increasing MM-bearing NOD/SCID mouse survival than that of EPI-MBs + mAb without UTMD or using EPI alone. Obviously, the combination of EPI-loaded MBs with conjugated ABCG2 mAb plus UTMD could target CSCs for ultimately a better treatment of MM. The target-specific CSC inhibition mediated by several indirect mechanisms may be involved in the MBs conjugated with $\mathrm{mAb}$ directly binding to the ABCG2 molecule on the surface of MM CSCs, shown in Fig. 3c, which could cause EPI delivery from the MBs to MM CSCs under ultrasound action. In addition, the mAb can partly block the ABCG2 efflux pumps and reserve EPI in MM CSCs for developing a cytotoxic effect on tumor cells as shown in Fig. 4c. This synergetic action resulted in MM growth inhibition.

We hypothesize that the inhibitory activity of EPIMBs + mAb combined with UTMD may be involved in induction of MM CSC apoptosis. This hypothesis was supported by the data from the immunohistochemistry assay. We found that the characteristic apoptosis molecules, such as Caspase-3, Bax, and TUNEL, were significantly increased in the tumor tissues of mice treated with the combination of EPI-MBs $+\mathrm{mAb}$ plus UTMD, whereas the inhibition of apoptosis and proliferation as well as promotion of vascularization molecules (PCNA, Bcl-2, and CD31) were respectively decreased compared with any other groups. Meanwhile, Western blot data showed that the expression of P-p65 and P-ІкB $\alpha, \quad$ NF- $\mathrm{kB}$ signal pathway-related molecules, was synchronously increased in tumor cells from the mice treated with EPI-MBs $+m A b$ plus UTMD, suggesting that the NF-kB signal pathway was activated. We presume that the EPI-loaded MBs conjugated with mAb were easily accumulated at tumor tissues, with thus more tumor cell uptake of EPI through perforations under ultrasound exposure, which resulted in increased DNA damage in tumor cells. DNA damage might activate the NF- $\mathrm{kB}$ signaling pathway [35].

We acknowledge that, in this study, the precise mechanisms underlying the effect of EPI-MBs + mAb plus UTMD remain unclear concerning the relevance of the apoptosis and NF- $\mathrm{kB}$ signaling pathways, particularly with respect to their impact on the synergetic therapeutic MM efficacy. Further investigation is therefore warranted. 


\section{Conclusion}

In conclusion, our findings reveal definitive evidence that the EPI-loaded MBs conjugated with ABCG2 mAb plus therapeutic ultrasound can target MM CSCs to develop an effective therapy for MM. The findings suggest an important strategy for a potential target ABCG2 molecule on CSCs for induction of MM CSC apoptosis.

\section{Abbreviations}

CSC: Cancer stem cell; EPI: Epirubicin; mAb: Monoclonal antibody; MB: Microbubble; MM: Multiple myeloma; UTMD: Ultrasound-targeted microbubble destruction

\section{Acknowledgements}

The authors appreciate Dr. Austin Smith at the Educational Testing Service, USA, for checking and editing our manuscript.

\section{Funding}

The study has been supported by the Nanjing Health Bureau Fundamental Research Foundation, Jiangsu Province, China (no. NJ2017005819), partly supported by the National Natural Science Foundation of China (no. 81572887), and partly supported by the Joint Research Project of Southeast University \& Nanjing Medical University (no.2242017K3DN41) and the Collaborative Innovation Center of Suzhou NanoScience and Technology.

\section{Authors' contributions}

JD designed, analyzed the data, and wrote the manuscript. FS was involved in the writing of the manuscript. FS, ML, JW, DW, MP, and MG performed the experiments. All authors read and approved the final manuscript.

\section{Ethics approval}

Animal experiments were conducted in accordance with the guidelines of the Animal Research Ethics Board of Southeast University. This ethics board also approved the animal studies.

\section{Consent for publication}

All authors have contributed to, read, and approved the final manuscript for submission.

\section{Competing interests}

The authors declare that they have no competing interests.

\section{Publisher's Note}

Springer Nature remains neutral with regard to jurisdictional claims in published maps and institutional affiliations.

\section{Author details}

${ }^{1}$ Department of Oncology, Zhongda Hospital, School of Medicine, Southeast University, Nanjing 210009, China. ${ }^{2}$ Department of Pathogenic Biology and Immunology, School of Medicine, Southeast University, 87\# Ding Jiaqiao Rd., Nanjing 210009, China. ${ }^{3}$ Department of Gynecology \& Obstetrics, Zhongda Hospital, School of Medicine, Southeast University, Nanjing 210009, China.

Received: 9 January 2018 Revised: 5 April 2018

Accepted: 23 April 2018 Published online: 21 May 2018

\section{References}

1. Matsui W, Huff CA, Wang Q, Malehorn MT, Barber J, Tanhehco Y, et al. Characterization of clonogenic multiple myeloma cells. Blood. 2004;103: 2332-6.

2. Waldschmidt JM, Simon A, Wider D, Müller SJ, Follo M, Ihorst G, et al. CXCL12 and CXCR7 are relevant targets to reverse cell adhesion-mediated drug resistance in multiple myeloma. J Haematol. 2017;79:36-49.

3. Kumar SK, Rajkumar V, Kyle RA, van Duin M, Sonneveld P, Mateos MV, et al. Multiple myeloma. Nat Rev Dis Primers. 2017;3:17046.

4. Dou J, Li Y, Zhao F, Hu W, Wen P, Tang Q, et al. Identification of tumor stem-like cells in a mouse myeloma cell line. Cell Mol Biol (Noisy-le-grand). 2009:55(Suppl):L1151-60

5. Matsui W. Perspective: a model disease. Nature. 2011;480:S58.
6. Ray A, Das DS, Song Y, Macri V, Richardson P, Brooks CL, et al. A novel agent SL-401 induces anti-myeloma activity by targeting plasmacytoid dendritic cells, osteoclastogenesis and cancer stem-like cells. Leukemia. 2017;31:2652-60.

7. Issa ME, Cretton S, Cuendet M. Targeting multiple myeloma cancer stem cells with natural products: lessons from other hematological malignancies. Planta Med. 2017;83:752-60.

8. Villa R, Cerroni B, Viganò L, Margheritelli S, Abolafio G, Oddo L, Paradossi G, et al. Targeted doxorubicin delivery by chitosan-galactosylated modified polymer microbubbles to hepatocarcinoma cells. Colloids Surf B Biointerfaces. 2013;110:434-42.

9. Geers B, De Wever O, Demeester J, Bracke M, De Smedt SC, Lentacker I. Targeted liposome-loaded microbubbles for cell-specific ultrasoundtriggered drug delivery. Small. 2013;9:4027-35.

10. Zhu F, Jiang Y, Luo F, Li P. Effectiveness of localized ultrasound-targeted microbubble destruction with doxorubicin liposomes in $\mathrm{H} 22$ mouse hepatocellular carcinoma model. J Drug Target. 2015;23:323-34.

11. Sorace AG, Warram JM, Umphrey H, Hoyt K. Microbubble-mediated ultrasonic techniques for improved chemotherapeutic delivery in cancer. J Drug Target. 2012;20:43-54.

12. Azmin M, Harfield C, Ahmad Z, Edirisinghe M, Stride E. How do microbubbles and ultrasound interact? Basic physical, dynamic and engineering principles. Curr Pharm Des. 2012;18:2118-34.

13. Wang Y, Bai WK, Shen E, Hu B. Sonoporation by low-frequency and low-power ultrasound enhances chemotherapeutic efficacy in prostate cancer cells in vitro. Oncol Lett. 2013;6:495-8.

14. Schatton T, Murphy GF, Frank NY, Yamaura K, Waaga-Gasser AM, Gasser $M$, et al. Identification of cells initiating human melanomas. Nature. 2008;451:345-9.

15. Turner JG, Gump JL, Zhang C, Cook JM, Marchion D, Hazlehurst L, Munster $P$, et al. ABCG2 expression, function, and promoter methylation in human multiple myeloma. Blood. 2006;108:3881-9.

16. Yang CP, Xiong F, Wang J, Dou J, Chen J, Chen D, et al. Anti-ABCG2 monoclonal antibody in combination with paclitaxel nanoparticles against cancer stem-like cell activity in multiple myeloma. Nanomedicine. 2014;9:45-60.

17. Abe M, Harada T, Matsumoto T. Concise review: defining and targeting myeloma stem cell-like cells. Stem Cells. 2014;32:1067-73.

18. Shi F, Yang F, He X, Zhang Y, Wu S, Li M, et al. Inhibitory effect of epirubicin-loaded lipid microbubbles with conjugated anti-ABCG2 antibody combined with therapeutic ultrasound on multiple myeloma cancer stem cells. J Drug Target. 2016;24:34-46.

19. Ghoroghchian PP, Frail PR, Susumu K, Blessington D, Brannan AK, Bates FS, et al. Near-infrared-emissive polymersomes: self-assembled soft matter for in vivo optical imaging. Proc Natl Acad Sci U S A. 2005;102:2922-7.

20. Kumar R, Belz J, Markovic S, Jadhav T, Fowle W, Niedre M, et al. Nanoparticlebased brachytherapy spacers for delivery of localized combined chemoradiation therapy. Int J Radiat Oncol Biol Phys. 2015;91:393-400.

21. Yang C, Xiong F, Dou J, Xue J, Zhan X, Shi F, et al. Target therapy of multiple myeloma by PTX-NPs and ABCG2 antibody in a mouse xenograft model. Oncotarget. 2015;6:27714-24.

22. Pu C, Chang S, Sun J, Zhu S, Liu H, Zhu Y, et al. Ultrasound-mediated destruction of LHRHa-targeted and paclitaxel-loaded lipid microbubbles for the treatment of intraperitoneal ovarian cancer xenografts. Mol Pharm. 2014;11:49-58.

23. Yang H, Cai W, Xu L, Lv X, Qiao Y, Li P, et al. Nanobubble-affibody: novel ultrasound contrast agents for targeted molecular ultrasound imaging of tumor. Biomaterials. 2015;37:279-88.

24. Dou J, Pan M, Wen P, Li Y, Tang Q, Chu L, et al. Isolation and identification of cancer stem-like cells from murine melanoma cell lines. Cell Mol Immunol. 2007:4:467-72.

25. Xia T, Kovochich M, Brant J, Hotze M, Sempf J, Oberley T, et al. Comparison of the abilities of ambient and manufactured nanoparticles to induce cellular toxicity according to an oxidative stress paradigm. Nano Lett. 2006;6:1794-807.

26. Asharani P, Low Kah Mun G, Hande MP, Valiyaveettil S. Cytotoxicity and genotoxicity of silver nanoparticles in human cells. ACS Nano. 2008;3:279-90.

27. Dimcevski G, Kotopoulis S, Bjånes T, Hoem D, Schjøtt J, Gjertsen BT, et al. A human clinical trial using ultrasound and microbubbles to enhance gemcitabine treatment of inoperable pancreatic cancer. J Control Release. 2016;243:172-81. 
28. Batrakova EV, Dorodnych TY, Klinskii EY, Kliushnenkova EN, Shemchukova OB, Goncharova ON, et al. Anthracycline antibiotics non-covalently incorporated into the block copolymer micelles: in vivo evaluation of anti-cancer activity. Br J Cancer. 1996;74:1545-52.

29. Wartlick F, Bopp A, Henninger C, Fritz G. DNA damage response (DDR) induced by topoisomerase II poisons requires nuclear function of the small GTPase Rac. Biochim Biophys Acta. 1833;2013:3093-103.

30. Yang X, Lin X, Zhong X, Kaur S, Li N, Liang S, et al. Double-negative feedback loop between reprogramming factor LIN28 and microRNA let-7 regulates aldehyde dehydrogenase 1-positive cancer stem cells. Cancer. 2010;70:9463-72.

31. Jones RJ. Cancer stem cells-clinical relevance. J Mol Med (Berl). 2009;87: 1105-10.

32. Cahu J, Bustany S, Sola B. Senescence-associated secretory phenotype favors the emergence of cancer stem-like cells. Cell Death Dis. 2012;3:e446.

33. Brayer J, Baz R. The potential of ixazomib, a second-generation proteasome inhibitor, in the treatment of multiple myeloma. Ther Adv Hematol. 2017;8:209-20

34. Ouyang L, Shen LY, Li T, Liu J. Inhibition effect of Oncostatin M on metastatic human lung cancer cells 95D in vitro and on murine melanoma cells B16BL6 in vivo. Biomed Res. 2006;27:197-202.

35. Ho WC, Dickson KM, Barker PA. Nuclear factor-kappaB induced by doxorubicin is deficient in phosphorylation and acetylation and represses nuclear factor-kappaB-dependent transcription in cancer cells. Cancer Res. 2005;65:4273-81.

Ready to submit your research? Choose BMC and benefit from:

- fast, convenient online submission

- thorough peer review by experienced researchers in your field

- rapid publication on acceptance

- support for research data, including large and complex data types

- gold Open Access which fosters wider collaboration and increased citations

- maximum visibility for your research: over $100 \mathrm{M}$ website views per year

At BMC, research is always in progress.

Learn more biomedcentral.com/submissions 